\section{Molecular Imaging of EGFR: It's Time to Go Beyond Receptor Expression}

TO THE EDITOR: In a recent issue of The Journal Nuclear Medicine, 2 interesting papers on PET detection of epidermal growth factor receptor (EGFR) in cancer were published $(1,2)$. Liu et al. reported the potential of ${ }^{11} \mathrm{C}$-PD153035 for the imaging of EGFR expression in humans, especially in non-small cell lung cancer (1). Tolmachev et al. reported that radiometal-labeled monomeric $Z_{\mathrm{EGFR}: 1907}$ is the preferable format of EGFR-specific Affibody (Affibody $\mathrm{AB}$ ) for imaging EGFR expression in mice with A431 cervical carcinoma xenografts (2).

The 2 articles differ from each other in several ways, such as the setting (humans and animals, respectively), the approach (labeled tyrosine kinase [TK] inhibitors and monoclonal antibody anti-EGFR, respectively), and the aims (metabolism/radiation dosimetry and imaging potential, respectively). Despite these relevant differences, both articles highlight an important issue for nuclear medicine and medical oncology research. The problem of detecting in vivo EGFR with a noninvasive approach is one of the most challenging in the selection of patients to receive EGFR inhibitors. However, some areas of discussion in these 2 papers need to be examined.

In brief, Lu et al. chose PD153035, a small-molecule TK inhibitor, labeled with ${ }^{11} \mathrm{C}$ as a promising PET tracer. PD153035 is a reversible EGFR inhibitor and has been considered the prototype of this class of tracer $(3,4)$. However, a preclinical study performed on tumor-bearing nude mice showed that the reversible compound may have a high non-tumor-specific uptake, probably bringing about a high competition with intracellular adenosine triphosphate at the binding site of the compound (5). However, the reason this study is of great interest is because it was the first such study performed on humans and opened the possibility of further clinical investigations. In Western countries, this aspect is relevant because the legislative process before a pharmaceutical drug can first be investigated in humans is lengthy and requires great effort. As a consequence, some promising novel tracers for EGFR imaging investigated in the United States or Europe still face extensive delays before being translated into human studies.

Tolmachev et al. chose the totally different approach of labeling the monoclonal antibody anti-EGFR. The strength of their study is that they used an Affibody that has a molecular weight lower than that of monoclonal antibodies, permitting high-contrast images of tumor receptor expression. The same approach had already shown successful results for HER2 imaging (6). However, some doubts still remain on the possibility of using it for imaging liver metastases or for metastatic sites near the kidneys.

During the last few years, several papers have been published on this topic and several advances have been made, but we believe that the development of the ideal PET tracer for EGFR imaging is still far distant (7-9). From an oncologic point of view, we need to discuss the actual usefulness of in vivo detection of EGFR expression in cancer today. As is well known, the most reliable clinical application is the prediction of anti-EGFR treatment response. However, during the last few years, advances in molecular predictive factors have

COPYRIGHT @ 2009 by the Society of Nuclear Medicine, Inc. been made. After the first evidence had been found by Lynch et al. and Paez et al. $(10,11)$, several papers showed that EGFR mutational status is a sensitive predictor of TK inhibitor activity in lung cancer. Furthermore, K-ras mutational status now represents the main available biomarker discriminating responders from nonresponders to anti-EGFR monoclonal antibodies in colorectal cancer and is the only one that should be translated into everyday clinical practice (12). Anti-EGFR drugs showed activity independently of total EGFR quantity; consequently, interest in the detection of receptor expression was lost. Moreover, we can suppose that the difficulties that emerged for EGFR imaging depended on the receptor amount, which was probably much too low to be detected in vivo. The approach of in vivo molecular imaging of membrane receptors may be more feasible and useful only in cases of receptor amplification, such as HER2 in breast cancer. As a consequence, we should pay more attention to both the activation of the receptor and its downstream signaling pathway, which are, respectively, influenced by EGFR and K-ras gene mutations. In fact, Pal et al. showed that the activation status of EGFR may be detected by in vivo imaging using PET probes that specifically bind only the adenosine triphosphate site of phosphorylated EGFR TK (13). Memon et al. showed that nude mice bearing xenografts of HCC827 cells harboring an in-frame deletion-mutation in exon 19 had the highest uptake of ${ }^{11} \mathrm{C}$-erlotinib (14). In both studies, the specific use of activated or mutated cell lines for the development of the animal model suggested that EGFR PET may be strictly dependent on the functional and mutational status of the receptor. Moreover, it was reported that the biologic features underlying the various EGFR mutations in non-small cell lung cancer are distinct and thus may confer different cellular properties or different TK activities and, consequently, different sensitivities to EGFR inhibitors (15). This variability should also be considered in the development of animal models and the design of chemical compounds as PET probes.

Currently, the added value of studying and using PET tracers for EGFR in clinical practice would be the possibility of obtaining information in vivo, in contrast to ex vivo molecular techniques, and of obtaining global information on all metastatic sites and with a repeatable approach during the progression of disease (16). Nevertheless, we would like to underline that the truly useful information is related to the cellular functional modifications underlying EGFR activation and not to EGFR expression.

In conclusion, over the near term, it would be more challenging to focus on imaging receptor function, which one can influence by mutations or by an aberrant downstream signaling pathway in order to reflect the complexity and variability of the EGFR pathway in human cancer. At any rate, the recent papers and all ongoing projects contribute to improving our understanding of this fascinating but also extremely difficult facet of nuclear medicine and medical oncology.

\section{REFERENCES}

1. Liu N, Li M, Li X, et al. PET-based biodistribution and radiation dosimetry of epidermal growth factor receptor-selective tracer ${ }^{11} \mathrm{C}$-PD153035 in humans. J Nucl Med. 2009;50:303-308.

2. Tolmachev V, Friedman M, Sandström M, et al. Affibody molecules for epidermal growth factor receptor targeting in vivo: aspects of dimerization and labeling chemistry. J Nucl Med. 2009;50:274-283. 
3. Fredriksson A, Johnström P, Thorell JO, et al. In vivo evaluation of the biodistribution of ${ }^{11} \mathrm{C}$-labeled PD153035 in rats without and with neuroblastoma implants. Life Sci. 1999;65:165-174.

4. Wang H, Yu J, Yang G, et al. Assessment of ${ }^{11} \mathrm{C}$-labeled-4-N-(3-bromoanilino)6,7-dimethoxyquinazoline as a positron emission tomography agent to monitor epidermal growth factor receptor expression. Cancer Sci. 2007;98:1413-1416.

5. Bonasera TA, Ortu G, Rozen Y, et al. Potential ${ }^{18} \mathrm{~F}$-labeled biomarkers for epidermal growth factor receptor tyrosine kinase. Nucl Med Biol. 2001;28:359-374.

6. Tolmachev V, Nilsson FY, Widström C, et al. ${ }^{111}$ In-benzyl-DTPA-ZHER2:342, an Affibody-based conjugate for in vivo imaging of HER2 expression in malignant tumors. J Nucl Med. 2006;47:846-853.

7. Pantaleo MA, Nannini M, Maleddu A, et al. Experimental results and related clinical implications of PET detection of epidermal growth factor receptor (EGFr) in cancer. Ann Oncol. 2009;20:213-226.

8. Mishani E, Abourbeh G, Eiblmaier M, Anderson CJ. Imaging of EGFR and EGFR tyrosine kinase overexpression in tumors by nuclear medicine modalities. Curr Pharm Des. 2008;14:2983-2998.

9. Gelovani JG. Molecular imaging of epidermal growth factor receptor expression-activity at the kinase level in tumors with positron emission tomography. Cancer Metastasis Rev. 2008;27:645-653

10. Lynch TJ, Bell DW, Sordella R, et al. Activating mutations in the epidermal growth factor receptor underlying responsiveness of non-small-cell lung cancer to gefitinib. $N$ Engl J Med. 2004;350:2129-2139.

11. Paez JG, Jänne PA, Lee JC, et al. EGFR mutations in lung cancer: correlation with clinical response to gefitinib therapy. Science. 2004;304:1497-1500.

12. Allegra CJ, Jessup JM, Somerfield MR, et al. American Society of Clinical Oncology provisional clinical opinion: testing for KRAS gene mutations in patients with metastatic colorectal carcinoma to predict response to antiepidermal growth factor receptor monoclonal antibody therapy. J Clin Oncol. 2009;27:2091-2096.

13. Pal A, Glekas A, Doubrovin M, et al. Molecular imaging of EGFR kinase activity in tumors with ${ }^{124} \mathrm{I}$-labeled small molecular tracer and positron emission tomography. Mol Imaging Biol. 2006;8:262-277.

14. Memon AA, Jakobsen S, Dagnaes-Hansen F, Sorensen BS, Keiding S, Nexo E. Positron emission tomography (PET) imaging with $\left[{ }^{11} \mathrm{C}\right]$-labeled erlotinib: a micro-PET study on mice with lung tumor xenografts. Cancer Res. 2009; 69:873-878.

15. Kancha RK, von Bubnoff N, Peschel C, Duyster J. Functional analysis of epidermal growth factor receptor (EGFR) mutations and potential implications for EGFR targeted therapy. Clin Cancer Res. 2009;15:460-467.

16. Pantaleo MA, Fanti S, Nannini M, et al. What oncologists need and require from nuclear medicine. Eur J Nucl Med Mol Imaging. 2008;35:1761-1765.

\section{Maria A Pantaleo Margherita Nannini Stefano Fanti Stefano Boschi Pier-Luigi Lollini Guido Biasco University of Bologna Bologna, Italy}

\section{DOI: 10.2967/jnumed.109.063909}

REPLY: We agree with Pantaleo et al. on the importance of developing novel molecular imaging agents that can provide information on epidermal growth factor receptor (EGFR) mutations to identify potential responders to EGFR-targeted therapeutics. We agree also that the value of wild-type EGFR expression as a predictive biomarker for anti-EGFR therapy in lung and colorectal cancer has not been demonstrated using common contemporary methods. However, the role of wild-type EGFR as a predictive biomarker for therapy of several malignancies has been shown using the existing detection methods. For example, a prospective study (1) has demonstrated that a high level of EGF expression can predict local-regional relapse after radiotherapy of head and neck squamous cell carcinomas. Another study (2) has proved the key role of high EGFR expression for selection of patients who may benefit from hyperfractionated accelerated radiotherapy of head and neck squamous cell carcinomas. High EGFR expression is also a predictive biomarker for a poor response to preoperative radiotherapy in advanced rectal carcinoma (3) and for tamoxifen treatment of early-stage breast cancer (4). These studies show that EGFR expression data may change patient management. In addition, clinical studies suggest that overexpression of EGFR is a prognostic biomarker in breast (5), prostate (6), and ovarian (7) cancers. Furthermore, downregulation of EGFR may serve as a rapid pharmacodynamic biomarker for antiHSP90 therapy as shown by Niu et al. (8).

Pantaleo et al. stated in a recent review article (9) that "The assessment of EGFR in ex vivo tumours specimens is still controversial for both methodological and biological reasons. EGFR was evaluated by immunohistochemistry (IHC) in most clinical studies and in clinical practice, but it is now well known that IHC is not an ideal method for EGFR detection for several factors...." Radionuclide molecular imaging may be combined with ex vivo detection of EGFR expression, adding the clear advantages of being global, minimally invasive, less sensitive to intratumoral heterogeneity of expression, and easily repeatable for following a patient. Therefore, radionuclide molecular imaging has the potential to become a powerful and convenient tool to fully assess the diagnostic value of EGFR overexpression in a broader spectrum of malignancies.

Thus, in vivo imaging of EGFR expression may provide important diagnostic information. We believe that radionuclide molecular imaging of EGFR expression has several potential clinical uses as an important complement to other diagnostic information.

\section{REFERENCES}

1. Ang KK, Berkey BA, Tu X, et al. Impact of epidermal growth factor receptor expression on survival and pattern of relapse in patients with advanced head and neck carcinoma. Cancer Res. 2002;62:7350-7356.

2. Bentzen SM, Atasoy BM, Daley FM, et al. Epidermal growth factor receptor expression in pretreatment biopsies from head and neck squamous cell carcinoma as a predictive factor for a benefit from accelerated radiation therapy in a randomized controlled trial. J Clin Oncol. 2005;23:5560-5567.

3. Giralt J, de las Heras M, Cerezo L, et al. The expression of epidermal growth factor receptor results in a worse prognosis for patients with rectal cancer treated with preoperative radiotherapy: a multicenter, retrospective analysis. Radiother Oncol. 2005;74:101-108.

4. Giltnane JM, Rydén L, Cregger M, Bendahl PO, Jirström K, Rimm DL. Quantitative measurement of epidermal growth factor receptor is a negative predictive factor for tamoxifen response in hormone receptor positive premenopausal breast cancer. J Clin Oncol. 2007;25:3007-3014.

5. Nieto Y, Nawaz F, Jones RB, Shpall EJ, Nawaz S. Prognostic significance of overexpression and phosphorylation of epidermal growth factor receptor (EGFR) and the presence of truncated EGFRvIII in locoregionally advanced breast cancer. J Clin Oncol. 2007;25:4405-4413.

6. Schlomm T, Kirstein P, Iwers L, et al. Clinical significance of epidermal growth factor receptor protein overexpression and gene copy number gains in prostate cancer. Clin Cancer Res. 2007;13:6579-6584.

7. Psyrri A, Kassar M, Yu Z, et al. Effect of epidermal growth factor receptor expression level on survival in patients with epithelial ovarian cancer. Clin Cancer Res. 2005;11:8637-8643.

8. Niu G, Cai W, Chen K, Chen X. Non-invasive PET imaging of EGFR degradation induced by a heat shock protein 90 inhibitor. Mol Imaging Biol. 2008;10:99-106.

9. Pantaleo MA, Nannini M, Maleddu A, et al. Experimental results and related clinical implications of PET detection of epidermal growth factor receptor (EGFr) in cancer. Ann Oncol. 2009;20:213-226.

\author{
Vladimir Tolmachev \\ Uppsala University \\ Uppsala, Sweden
}

DOI: 10.2967/jnumed.109.064188 